\title{
EFFECT OF DAIRY PRODUCTS ON OXIDATIVE STRESS IN TYPE 2 DIABETIC PATIENTS: A RANDOMIZED CONTROLLED CLINICAL TRIAL
}

\author{
Parvin Mirmiran , Somayeh Hosseinpour-Niazi , Fereidoun Azizi \\ Department of Clinical Nutrition and Dietetics, Faculty of Nutrition Sciences and Food Technology, National Nutrition \\ and Food Technology Research Institute, Shahid Beheshti University of Medical Sciences, Tehran, Iran
}

mirmiran@endocrine.ac.ir; parvin.mirmiran@gmail.com

\section{BACKGROUND AND AIMS}

Some investigations suggest an important role for the types and numbers of servings of dairy products in controlling the risk of diabetes and its complications. The aim of this study is to investigate and compare the effect of inclusion of 2-3 or 4-5 servings of dairy products, ( milk and yogurt) on oxidative stress among type 2 diabetic patients.

\section{METHODS}

Diabetic patients with a diagnosis of diabetes as defined by fasting blood glucose concentrations $\geq 126$ $\mathrm{mg} / \mathrm{dl}$ or using oral glucose lowering medications and having a stabilized status for 3 months were recruited from the clinic of Taleghani hospital, Tehran, Iran.

Criteria included habitual intake of one portions of dairy products per day, age $30-50$ years, being nonsmokers, body mass index $25-30 \mathrm{~kg} / \mathrm{m}^{2}$, not currently receiving insulin therapy and not having cardiac, hepatic or renal function disorders. Ninety-one diabetic patients were randomly assigned to one of the three following intervention groups: Group A $(<1$ serving of dairy products), group B (2 to 3 servings), and group $\mathrm{C}$ (4 to 5 servings) over a period of 8 weeks. Biomarkers of oxidative stress including Serum fasting glucose, Serum total antioxidant capacity, catalase activity, malondialdehyde (MDA) were measured at baseline and after the 8-week intervention

\section{RESULTS}

Of the 91 diabetic patients who completed the study, $58 \%$ were female, with a mean age $57.7 \pm 8.9$ years. Based on 2-day diet records every week during the study, participants in $\mathrm{A}, \mathrm{B}$, and $\mathrm{C}$ groups consumed $0.9,2.8$ and 4.9 servings of dairy products per day. There was no significant difference in energy intake, macronutrients and total fiber between intervention groups. MDA in group $\mathrm{C}$ with 4 to 5 servings of dairy products per day was reduced significantly compared to both group B with 2 to 3 serving per day $(-1.5 \pm 2.7)$ and the controls $(0.4 \pm 4.1)$. No significant changes were found in FBS, TAC, CAT, and SOD after the three interventions

Table. Means of MDA at baseline and after 8 weeks of intervention in type 2 diabetic patients

\begin{tabular}{|c|c|c|c|c|}
\hline & Group A & Group B & Group C & P value \\
\hline \multicolumn{5}{|l|}{ FBS (mg/dl) } \\
\hline Baseline & $138 \pm 4.2$ & $139 \pm 4.2$ & $139 \pm 4.2$ & 0.82 \\
\hline End of trial & $140 \pm 4.3$ & $141 \pm 4.3$ & $140 \pm 4.3$ & 0.98 \\
\hline \multicolumn{5}{|l|}{ MDA ( $\mu \mathrm{mol} / \mathrm{l})$} \\
\hline Baseline & $7.1 \pm 3.0$ & $7.7 \pm 2.3$ & $6.6 \pm 2.8$ & 0.29 \\
\hline End of trial & $7.5 \pm 0.5$ & $6.1 \pm 0.4$ & $5.5 \pm 0.4$ & 0.03 \\
\hline \multicolumn{5}{|l|}{ TAC (mU/ml) } \\
\hline Baseline & $9.2 \pm 0.9$ & $9.9 \pm 0.9$ & $9.6 \pm 0.4$ & 0.53 \\
\hline End of trial & $10.5 \pm 1.5$ & $10.8 \pm 1.5$ & $10.4 \pm 1.5$ & 0.66 \\
\hline \multicolumn{5}{|l|}{ CAT (U/ml) } \\
\hline Baseline & $77.0 \pm 14.8$ & $75.6+15.6$ & $79.9 \pm 17.6$ & 0.49 \\
\hline End of trial & $87.5 \pm 14.9$ & $86.8 \pm 15.7$ & $86.2 \pm 17.9$ & 0.95 \\
\hline \multicolumn{5}{|l|}{ SOD (U/ml) } \\
\hline Baseline & $168 \pm 16.1$ & $172 \pm 16.3$ & $164 \pm 16.4$ & 0.38 \\
\hline End of trial & $166 \pm 16.1$ & $164 \pm 16.1$ & $156 \pm 16.3$ & 0.78 \\
\hline
\end{tabular}

CONCLUSIONS: Consumption of dairy products including milk and yogurt, $\geq 4$ servings per day, reduced MDA concentration, however consumption $\leq 3$ serving per

day had no significant effect on oxidative stress among diabetic patients. 